\title{
Tessellated Scale, Ecualymnatus tessellatus (Signoret) (Insecta: Homoptera: Coccidae) ${ }^{1}$
}

G.W. Dekle ${ }^{2}$

\section{Introduction}

Tessellated or palm scale is a soft scale that was first described in France by V. Signoret in 1873 from specimens collected on a palm he reported as Caryota ursus. The genus Eucalymnatus, according to Borchsenius (1957), contains 17 species; two species are Ethiopian in origin and the remainder neotropical. Borchsenius (1957) considers E. tessellatus (Sign.) probably South American in origin and disseminated by man to all parts of the world on its cultivated hosts.

\section{Synonymy}

Lecanium tessellatum Signoret, 1873; Eucalymnatus tessellatus, Cockerell \& Parrott, 1901; Eucalymnatus, Lindinger, 1943.

\section{Distribution}

In Florida, Division of Plant Industry collections include specimens from the following counties: Alachua, Baker, Brevard, Broward, Charlotte, Clay, Collier, Dade, Dixie, Duval, Escambia, Flagler, Glades, Gulf, Hendry, Hernando, Highlands,
Hillsborough, Indian River, Jefferson, Lake, Lee, Leon, Manatee, Marion, Martin, Monroe, Nassau, Orange, Palm Beach, Pasco, Pinellas, Polk, Putnam, St. Johns, St. Lucie, Sarasota, Seminole, and Volusia.

\section{Description}

The adult female is oval, opaque, reddish brown to dark brown, and 4 to $5 \mathrm{~mm}$ in length. The apex is angulate and the posterior broadly rounded. The dorsal surface appears checkered or cellular in pattern when examined with the aid of a 10-power hand lens or magnifying glass. A median, longitudinal, smooth ridge divides the cellular pattern on the dorsal surface. The cells formed nearer the ridge appear concave, progressively becoming flattened outwardly to the larger, flat, marginal cells.

\section{Hosts}

Achras zapota L. (sapodilla), Ajuga reptans L. (bugle weed), Allamanda neriifolia Hook., Alocasia sp., Alpinia speciosa (Wendl.) Schum. (shell ginger), Annona muricata L. (soursop), A. reticulata L. (common custard-apple, bullocks-heart), Anthurium andraeanum Lind., A. magnificum Lind.,

1. This document is EENY-090 (originally published as DPI Entomology Circular No. 138), one of a series of Featured Creatures from the Entomology and Nematology Department, Florida Cooperative Extension Service, Institute of Food and Agricultural Sciences, University of Florida. Published: June 1999. This document is also available on Featured Creatures Website at http://creatures.ifas.ufl.edu. Please visit the EDIS Website at http://edis.ifas.ufl.edu. Additional information on these organisms, including many color photographs, is available at the Entomology and Nematology Department WWW site at http://entnemdept.ifas.ufl.edu/.

2. G.W. Dekle, Florida Department of Agriculture and Consumer Services, Division of Plant Industry, Gainesville, FL.

The Institute of Food and Agricultural Sciences is an equal opportunity/affirmative action employer authorized to provide research, educational information and other services only to individuals and institutions that function without regard to race, color, sex, age, handicap, or national origin. For information on obtaining other extension publications, contact your county Cooperative Extension Service office. Florida Cooperative Extension Service/Institute of Food and Agricultural Sciences/University of Florida/Christine Taylor Waddill, Dean. 


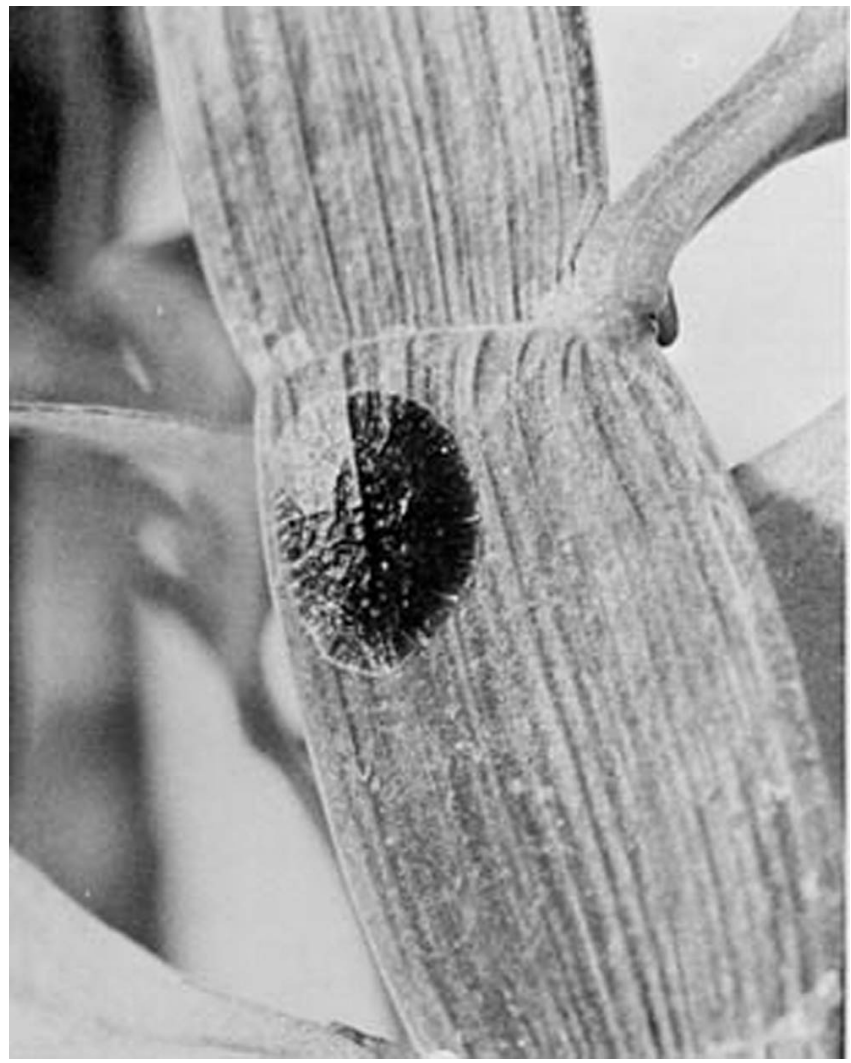

Figure 1. A female tessellated scale, Eucalymnatus tessellatus (Signoret), on host. Credits: Division of Plant Industry

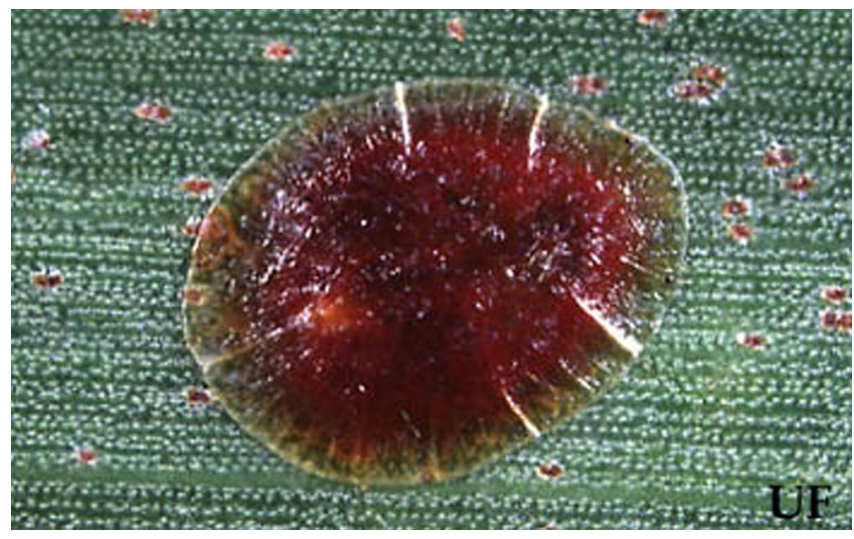

Figure 2. Tessellated scale, Eucalymnatus tessellatus (Signoret). Credits: Paul. M. Choate, University of Florida

Archontophoenix alexandrae Wendl. \& Drude (alexander palm), Ardisia escallonioides Schiede \& Deppe (marlberry), A. pickeringia Torr. \& Gray, Areca catechu L. (betelnut palm), Arecastrum romanzoffianum Becc. (queen's palm), Asplenium nidus L. (bird's nest fern), Baccharis angustifolia Michx. (groundsel), Bischofia javanica Toog., Brunsfelsia sp. (lady-of-the-night), cactus (unknown), Callistemon spp. (bottlebrush), Calocarpum sapota Merr. (sapota), Calophyllum sp. (beauty-leaf), Carissa sp., Caryota mitis Lour. (fish-tail palm), C. urens L. (wine-palm), Cestrumsp. (jessamine), Chamaedorea elegans Mart. (neanthe bella palm, dwarf palm, parlor palm), C. tenella $\mathrm{H}$. Wendl., Chrysalidocarpus lutescens Wendl. (areca palm), Chrysophyllum cainito L. (star-apple), Chrysophyllum sp. (satinleaf), Cinnamomum cassia Nees \& Eberm. (cassia bark tree), Cinnamomum sp. (cinnamon), Cleyera album Wendl. \& Drude, Dieffenbachia sp., Diospyros ebenaster Retz., Dipholis salicifolia (L.) A. DC. (willow bustic), Dizygotheca elegantissima (Veitch) Vig. \& Guill. (aralia), Dracaena sp., Elaeocarpus cyaneus Sims, Ervatamia coronaria Stapf. (crape-jasmine), Eugenia hookeriana Hort. (Australian brush cherry), Euphoria longana Lam. (longan, lungan), Eurya sp., Fatshedera lizei (Cochot) Guill., Fatsia sp., Feijoa sellowiana Berg., Ficus jacquiniaefolia A. Rich (jacquinia fig), F. rubiginosa Vent. (rusty fig), Gardenia jasminoides Ellis (cape jasmine), Genipa clusiaefolia (Jacq.) Griseb. (seven-year apple), Gerbera jamesonii Bolus (African daisy), Gordonia lasianthus (L.) Ellis (loblolly bay), Hedera sp. (English ivy, ivy), Hedychium sp. (gingerlily), Homalocladium platycladium Bailey (ribbon plant, ribbon bush, tapeworm plant), Ilex cassine $\mathrm{L}$. (Florida holly, dahoon holly, yaupon holly), I. cornuta Lindl. (Chinese holly), I. cornuta Lindl. 'Burfordii' (Burford holly), I. glabra (L.) A. Gray (gallberry), I. latifolia Thunb. (lusterleaf holly), Ixora parviflora Vahl. (giant ixora), Ixora sp. (white ixora), Jasminum sp. (jasmine), Kentia belmoreana F. Muell., Kigelia pinnata DC. (sausage tree), Laurus nobilis L. (sweet bay tree), Litchi chinensis Sonner (lychee), Lucuma nervosa A. DC. (canistel, eggfruit), Macrozamia peroffskyana Miq. (a cycad), Magnolia virginiana $\mathrm{L}$. (white bay), Mangifera indica $\mathrm{L}$. (mango), Mascarena sp., Melaleuca leucadendron L. (punk tree, cajeput), Mimusops emarginata L. (wild sapodilla), M. roxburghiana Wight (bulletwood), Muehlenbeckia sp. (inch plant, maidenhair vine, wire plant), Musa sp. (banana), Myrtus sp., Nerium sp. (oleander), Ochrosia sp. (kopsia), Ocotea catesbyana (Michx.) Sarg., Peltandra virginica Kunth (green arrow-arum), Persea americana Mill. (avocado), Philodendron sp., Phoenix roebeleni O'Brien (pigmy date palm), Phoradendron flavescens Nutt. (mistletoe), Pilea sp. (aluminum plant), 
Ptychosperma elegans (R. Br.) Blume (solitaire palm), Rapanea guaynensis Aubl., Rhapis excelsa (Thunb.) Henry (lady palm), Rhaphidophora decursiva Scott, Rhodomyrtus sp. (downy myrtle), Schinus terebinthifoius Raddi (Brazilian pepper tree), Scindapsus sp. (pothos), Stigmaphyllon sp., Strobilanthes sp., Syzgium jambos Alston (rose apple), Tetrapanax papyriferus Koch (Chinese rice paper plant), Thevetia peruviana (Pers.) Schum. (luckynut), Thunbergia sp., Trachycarpus fortunei $\mathrm{H}$. Wendl. (windmill palm), Trevesia palmata Vis. 'Micholitzii' (devil's tongue) and Viburnum odoratissimum Ker.

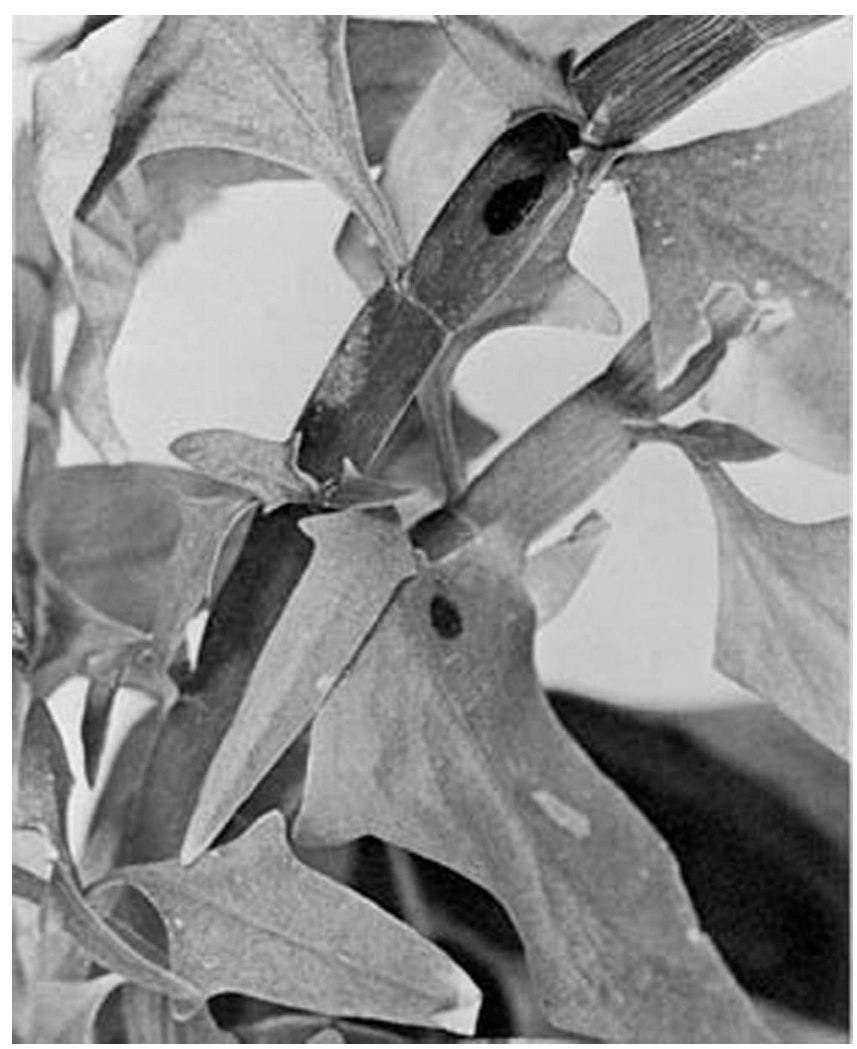

Figure 3. Tessellated scale, Eucalymnatus tessellatus (Signoret), on ribbon bush, Homalocladium platycladium Bailey. Credits: Division of Plant Industry

\section{Economic Importance}

Tessellated scale is primarily a leaf-infesting soft scale and a greenhouse pest. In South Florida, it is frequently found out-of-doors on palms, crape-jasmine, and mango. Infestations are often of economic importance in commercial nurseries whenever pest management programs are neglected.

\section{Management}

Insect Management Guide for Fruit

Insect Management Guide for Ornamentals

\section{Selected References}

Borchsenius, N.S. 1957. Fauna of USSR, Homoptera, Coccidae (in Russian). Akad. Nauk Zool. Inst. (n.s. 66) 9: 1-493.

Dietz, H.T., and H. Morrison. 1916. The Coccidae or scale insects of Indiana. 8th Ann. Rpt. State Ent. Indiana 8: 195-321.

Merrill, G.B. 1953. A revision of the scale insects of Florida. Florida State Plant Board Bull. 1: $1-143$.

Sanders, J.G. 1909. The identity and synonymy of some of our soft scale-insects. J. Econ. Ent. 2: 428-448.

Signoret, V. 1873. Essai sur les cochenilles ou gallinsectes. Ann. Soc. Ent. France (Ser. V) 3: 395-448.

Zimmerman, E.C. 1948. Insects of Hawaii, vol. 5, Homoptera: Sternorhyncha. Univ. of Hawaii Pr., Honolulu. 464 p. 